\title{
Minimum Data Set for Cystic Fibrosis Registry: a Case Study in Iran
}

\author{
Leila R Kalankesh', 2, Saeed Dastgiri' ${ }^{2,}$, Mandana Rafeey ${ }^{4,5}$, Narmin Rasouli', Leila Vahedi ${ }^{4}$ \\ 'School of Management and Medical Informatics, Tabriz University of Medical Sciences \\ ${ }^{2}$ Health Services Management Research Center, Tabriz University of Medical Sciences \\ ${ }^{3}$ Department of Community and Family Medicine, School of Medicine, Tabriz University of Medical Sciences \\ ${ }^{4}$ Liver \& Gastrointestinal Research Center, Tabriz University of Medical Sciences \\ ${ }^{5}$ Department of Pediatrics, Children Hospital, Tabriz University of Medical Sciences
}

Corresponding author: Dr Leila R Kalankesh, PhD, School of Management and Medical Informatics, Tabriz University of Medical Sciences, Iran, Email: Irkalankesh@tbzmed.ac.ir

\begin{abstract}
Background: over the last 25 years several national registries of CF have been set up. Such systems can be very useful in providing an integrated resource for improving patient care and conducting research on the disease. Minimum Data Set is a common set of data items that should be used to collect and report data in the registry. The principal aim of this research was to determine minimum data set for the CF registry in north-west of Iran. Methods: data items collected by several selected registries of cystic fibrosis were studied and an initial set of data was selected by the researchers. A group of experts including epidemiologists, pediatricians, and CF specialists were asked to review the proposed data elements and score them based on their importance by using a nine-point Likert scale. The items scored as important or highly important by more than $50 \%$ of the experts, were included in final list of minimum data set. Availability of data was evaluated through reviewing medical records of 144 patients hospitalized in Children Hospital located in Tabriz. Results: overall six classes of data ( 46 items) were identified in the selected registry systems for cystic fibrosis: patient demographics, administrative data, survival status, diagnostic procedures, genetic and clinical manifestations, and therapeutics. Thirty two data elements from all six categories of data were approved by the experts as the minimum data set for cystic fibrosis registry system. Availability of data in administrative category and survival class was 100 percent. Collecting data on medications was feasible in $100 \%$ of the cases as well. In class of demographic data, accessibility of patient name, age, gender, place of birth, and date of birth was 100 percent. In group of diagnostic procedures, partial availability of data was found for sweat test and genetic test. No data was found on the antenatal screening, exercise tolerance test, and glucose tolerance test. Conclusion: this work can be considered as a first step toward establishing CF registry system in Iran. Minimum data set can be also useful in designing electronic registry or electronic patient records for those suffering from CF toward integration of their fragmented records across continuum of the health care system in order to improve quality of shared patient care. Keywords: minimum data set, registry, data elements, cystic fibrosis, and core data set.
\end{abstract}

\section{INTRODUCTION}

Cystic fibrosis is an autosomal recessive disease and a hereditary disease of mucus and sweat glands caused by a CF transmembrane regulator defect that mainly affects the respiratory and gastrointestinal systems, leading to progressive disability $(1,2)$. According to WHO, 1 in 2000-3000 newborns is affected by CF across the Europe; in north America the incidence has been reported to be 1 in 3500; no accurate data are available in Africa; in middle east various incidence rates have been reported ranging from 1 in 2560 to 1 in 15876 (3). However these statistics differ from country to country. For example the incidence reported for Ireland, UK, Belgium, Spain are $2.98,1.37,1.03,0.546$ in ten thousand respectively (4). No data are available for Iran in the report of CF by WHO (3). However there are a few papers reporting different statistics of CF in different regions of Iran. For instance, while no confirmed case of CF has been reported in southern Iran (5), there is a report from north-west of Iran on CF prevalence of 7.98 in 100 thousand during the 5-year period (2004-2008)(6) in addition to a study reported growth pattern and nutritional intake of 34 infants with CF in East Azerbaijan province (7).
Moreover, spectrum of CFTR gene mutations in 200 Iranian Azeri Turkish Patients with Cystic Fibrosis has been examined and reported in another study (8).

Registries are considered essential tools designed to measure all health-related aspects of cystic fibrosis (CF) and to compare clinical data from different centers and countries. Over the last 25 years, several national registries of CF have been set up (9). European Committee of Experts on Rare Diseases emphasizes the importance of the registry system on rare diseases such as CF. This is due to the fact that the registry system can be very useful in cases of rare disease such as cystic fibrosis in providing an integrated resource for improving patient care and research on the disease $(10,11)$.

Minimum Data Set is a common set of data items that should be used to collect and report data in the registry (12). To best of our knowledge, no research has been undertaken so far in order to identify minimum data set for cystic fibrosis in Iran. This paper represents our attempt to identify minimum data set for cystic fibrosis registry system in Northwest of Iran. 


\section{METHODS}

To determine the minimum data set required for establishing registry system of cystic fibrosis, the systems of selected countries including Netherlands (13), the US (14), Ireland (15), UK (16), France (17, 18), Australia (19), Brazil (20), Canada (21), Belgium (22) and New Zealand (23) as well as the registry system of European Cystic Fibrosis Society (24) were studied.

Considering the commonalities and differences observed among data elements of the studied registry systems and regional demands, an initial set of data elements was proposed by the researchers. Then a group of multidisciplinary experts consisted of epidemiologists, pediatricians, and CF specialists were asked to review and score the initial set based on their importance by using a nine-point Likert scale ranging from 1 to 9 where 1 referred to concept of "no important for inclusion in MDS" and 9 indicated the statement of "highly important for inclusion in MDS". Data elements that were scored as important or highly important by more than 50 percent of the experts were included in the final minimum data set.

In next stage of the research, availability of data on the agreed minimum data set was evaluated through reviewing the medical records of 144 patients (191 episode of care) hospitalized with diagnosis of cystic fibrosis in Tabriz children hospital from 2009 to 2014.

\section{RESULTS}

\subsection{Identification of initial data elements}

Overall six classes of data (including 46 data items) were identified in the selected cystic fibrosis registry systems: patient demographics, administrative data, survival status, diagnostic procedures, genetic and clinical manifestations, and therapeutics. Overall 34 data elements were determined as an initial set as follows:

- Patient demographics: name, gender, age, weight, height, BMI, date of birth, age at diagnosis, job, place of birth, socioeconomic status, and time off from work or school

- Administrative data: CF centre identification code, patient identification code, year of follow-up, cost of hospitalization, frequency of hospitalization per year, and date of encounter

- Survival data: death date(if any), and cause of death

- Diagnostic procedures: sweat test, genetic test, antenatal screening, exercise tolerance test, and glucose tolerance test

- Disease genotype and clinical manifestation: CF genotype, signs and symptoms, FEV1, FVC, and complications

- Therapeutics: medications, and organ transplantation

\subsection{Agreed minimum data set}

Data elements scored as highly important or important at least by more than 50 percent of the experts are presented in table 1 .

\begin{tabular}{lll}
\hline More than 75\% & $51-75 \%$ & $51-75 \%$ \\
\hline Date of birth & Name & Date of encounter \\
\hline Age at diagnosis & Gender & Organ transplantation \\
\hline Cause of death & Age & $\begin{array}{l}\text { Time off from work or } \\
\text { school }\end{array}$ \\
\hline CF complications & Weight & Follow-up year \\
\hline FEV1 & Height & Glucose tolerance test \\
\hline FVC & BMI & Exercise tolerance test \\
\hline $\begin{array}{l}\text { Frequency of hospitaliza- } \\
\text { tion per year }\end{array}$ & $\begin{array}{l}\text { Patient identifica- } \\
\text { tion code }\end{array}$ & Medications \\
\hline Sweat test & $\begin{array}{l}\text { CF centre identifi- } \\
\text { cation code }\end{array}$ & Antenatal screening \\
\hline Signs and symptoms & $\begin{array}{l}\text { Socio-economic } \\
\text { status }\end{array}$ & Cost of hospitalizations \\
\hline & Birth Place & Genetic test \\
\hline & Death date & Genotype \\
\hline
\end{tabular}

Table 1. Minimum data set approved by the experts for CF registry

\subsection{Availability of the data}

As figure 1 depicts availability of data in administrative category and survival class is 100 percent. Collecting data on medications was feasible in $100 \%$ of the cases as well. In class of demographic data, availability of patient name, age, gender, place of birth, and date of birth was 100 percent. In group of diagnostic procedures, partial availability of data was observed for sweat and genetic test. However no data were found on antenatal screening, exercise tolerance test, and glucose tolerance test.

\section{DISCUSSION}

This paper represents the first attempt undertaken to develop minimum data set for registry system of cystic fibrosis in Northwest of Iran. Comparative study of the registry systems provided a foundation for defining the initial MDS. There were six different classes of data elements including patient

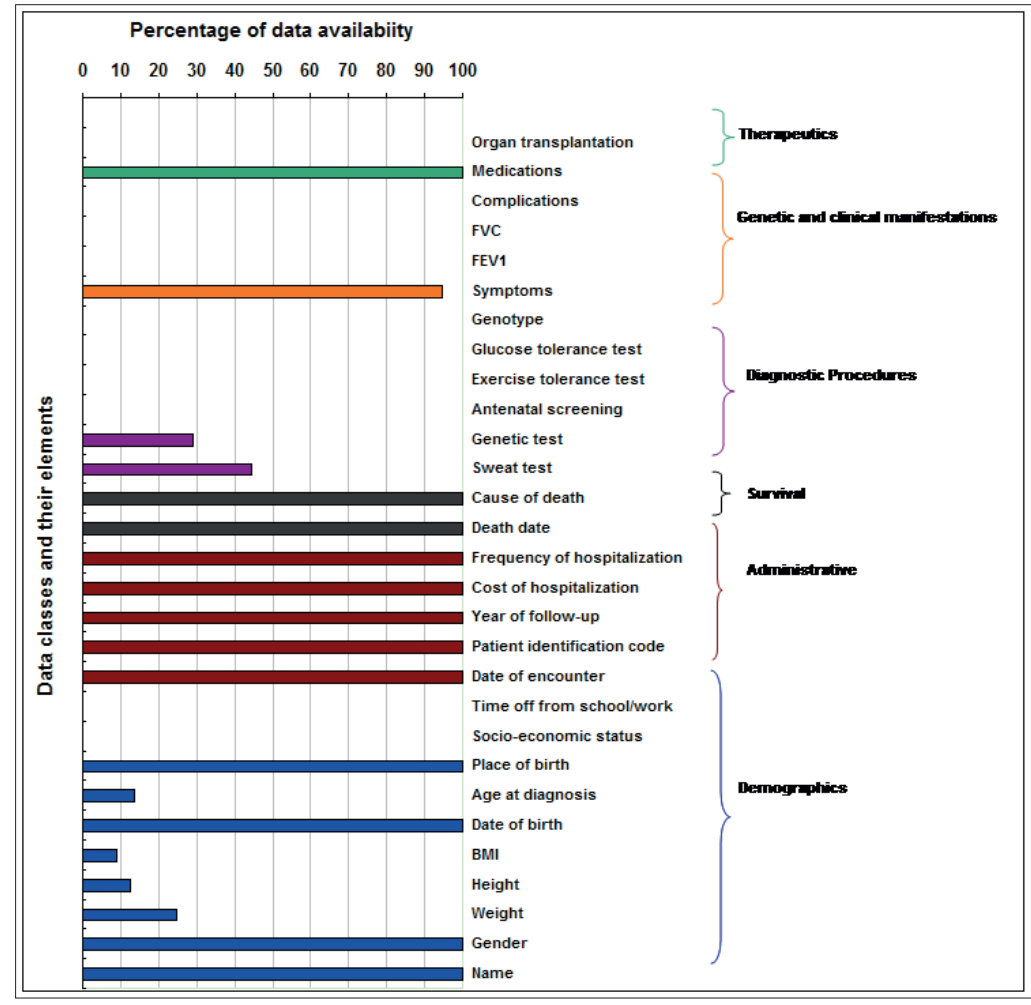

Figure 1. Percentage of the data availability for CF registry in patient records 
demographics, administrative data, survival data, diagnostic procedures, genetic and clinical manifestation, and therapeutics. Majority of data elements from all six classes were evaluated as highly important or important by the experts. This is similar to the classes of data determined as minimum data set for athlete health records (25), physiotherapy (26), breast cancer (27), orthopedic injuries in Iran (28), nursing (29) by their related experts. This is also in line with information classes found in data content residing in GP systems (30). This can reflect the importance of these categories in different domains of health care system. This can be also due to the fact that minimum data set can be used in variety of use cases: for patient care in individual level; for assessment of system and care provider in organizational level; and for national and international comparisons in aggregated level (12). Moreover minimum data set can contribute to realization of conceptual interoperability throughout all these levels (31).

Reviewing medical records of patients with CF revealed availability of the data in real world. Majority of data in administrative and demographic categories were highly available in the patient records. This is viable as the data items such as patient identification code, patient name and encounter date are important for linking records from multiple resources (32) or data items such as age, gender,or birth place can be used for standard sociodemographic comparisons or reports as they are among high priority data items determined by CF data network (33). However it should be noted that patient identification code used in the medical records could only identify patient in the hospital not throughout the entire health system. Therefore the unique patient identification number with capability of recognizing patient across the entire health system is the missing element.

High priority given by the experts on date and cause of death and their full availability are in consistent with legal enforcement for recording such data (34). Medication data were found to be available in 100 percent of the cases. This is not surprising as these data are at the core of the direct patient care.

Partial availability of data for sweat test and genetic test was observed. Although sweat test is done for diagnosing all cases, and genetic test is carried on for most of the patients, but in most cases it is conducted in outpatient services and clinics that are not connected with the inpatient services and their results are usually kept by patient's family. This may also be attributed to lack of linkage or integration among different information silos across CF care related centers, including the laboratories or clinics. This may also reflect lack of appropriate data flow. Use of unique patient identifier across the whole continuum of health system can facilitate data flow and integration of patient care.

Despite high priority given to data items on diagnostic tests such as antenatal screening, exercise tolerance test and glucose tolerance test, no data was found on them in the hospital patient records. It should be noted that the problem with glucose tolerance occurs after age of 10 while all cases in this study are neonates and infants when they are diagnosed or admitted to the hospital. Exercise tolerance test is not a routine test and in case it is done, its data is not entered into hospital records as it is undertaken in private clinics. Antenatal screening is not also done because there is no precise statistic of patients with
CF in Iran. Unavailability of data on FEV1 and FVC is due to the fact that respiratory tests are not taken place in hospital but in private ambulatory services and clinics.

Lack of data on organ transplant is not surprising as the lung transplant for patients with CF has just newly introduced in Iran.

Finding about data availability in this research was restricted to children hospital records. Other resources in genetic laboratory centers, or clinics, or other related centers and hospitals were not studied. Lack of unique patient identifier across the entire health system seems to be the most important limit in integrating the related information resources. In addition lack of coding practice for ambulatory and outpatient health services makes it unfeasible to locate and retrieve data residing in the related centers.

\section{CONCLUSION}

This study presents minimum data set required for establishing cystic fibrosis registry in Northwest of Iran. Minimum data set can be also useful in designing electronic patient records or registry for those suffering from CF toward integration of their fragmented records across continuum of the health care system and for the shared patient care.

\section{Acknowledgement}

This paper is derived from MSc thesis, financially funded by School of Management and Medical Informatics at Tabriz University of Medical Sciences.

\section{CONFLICT OF INTEREST: NONE DECLARED.}

\section{REFERENCES}

1. Online Mendelian Inheritance in Man. Cystic Fibrosis. 2014; Available from: http://www.omim.org/entry/219700.

2. Marcdante K, Kliegman RM. The Respiratory System. Nelson Essentials of Pediatrics: Elsevier Health Sciences; 2014.

3. WHO. The molecular genetic epidemiology of cystic fibrosis Genoa; 2002.

4. Farrell PM. The prevalence of cystic fibrosis in the European Union. Journal of Cystic Fibrosis. 2008; 7(5): 450-453.

5. Karjoo M, Bahremand M, Mihandoust G. Cystic fibrosis in Southen Iran. Journal of Tropical Pediatrics. 1984;30(4): 195-196.

6. Dastgiri S, Bonyadi M, Mizaiee T. Genetic abnormalities in East Azarbaijan province. Urmia Medical Journal. [Research]. 2011 1389; 21(4): 339-346.

7. Rafeey M, Pourabdollahi P, Ghaemmaghami SJ, Samsamy M, Pourhossein D, Nasiri A. Growth pattern and nutritional intake in children with cystic fibrosis: comparison with normal children in East Azerbaijan-Iran. Urmia Medical Journal. 2010; 20(4): 278-283.

8. Bonyadi M, Omrani O, Rafeey M, Bilan N. Spectrum of CFTR gene mutations in Iranian Azeri Turkish patients with cystic fibrosis. Genet Test Mol Biomarkers. 2011;15(1-2): 89-92.

9. Salvatore D, Buzzetti R, Baldo E, Forneris MP, Lucidi V, Manunza D, et al. An overview of international literature from cystic fibrosis registries. Part Disease incidence, genotype/phenotype correlation, microbiology, pregnancy, clinical complications, lung transplantation, and miscellanea. Journal of Cystic Fibrosis. 2011; 10: 71-85.

10. Rare Diseases Task Force. Patient registration in the field of rare disease; 2011. 
11. Viviani L, Zolin A, Mehta A, Olesen HV. The european cystic fibrosis society patient registry: valuable lessons learned on how to sustain a disease registry. Orphanet journal of rare diseases. 2014; 9: 81 .

12. Kowal PR, Wolfson LJ, Dowd JE. creating a minimum data set on ageing in subsaharan Africa. Southern African Journal of Gerontology. 2000; 9(2): 18-23.

13. Netherlands Cystic Fibrosis Registry. Available from: http:// www.cfonderzoek.nl/cf-registratie.

14. United States Cystic Fibrosis Patient Registry. Available from: http://www.cff.org/LivingWithCF/ CareCenterNetwork/PatientRegistry.

15. The Cystic Fibrosis Registry of Ireland. Available from: http:// www.cfri.ie.

16. United Kingdom Cystic Fibrosis Registry. Available from: http://www.cysticfibrosisdata.org/data-registry/united-kingdom-cystic-fibrosis-registry.

17. French Cystic Fibrosis Registry. Available from: http://www. vaincrelamuco.org /ewb_pages/e/english-version.php?lang=en

18. Eurodis. French Cystic Fibrosis Registry. Berlin; 2006.

19. Australian Cystic Fibrosis Data Registry. Available from: http:// www.cysticfibrosis.org.au/cfa/data-registry.

20. Brazilian Cystic Fibrosis Registry. Available from: http://www. gbefc.org.br.

21. Canadian Cystic Fibrosis Registry. Available from: http:// www.cysticfibrosis.ca/cf-care/cf-registry.

22. The Belgian Cystic Fibrosis Registry. Available from: https:// www.wiv-isp.be/epidemio/epien/index20.htm.

23. New Zealand Cystic Fibrosis Registry. Available from: http:// www.cfnz.org.nz.

24. ECFS Patient Registry. Available from: http://www.ecfs.eu/ projects/ecfs-patient-registry/intro.
25. Safdari R, Dargahi H, Halabchi F, Shadanfar K, Abdolkhani R. A comparative study of the athlete health records' minimum data set in selected countries and presenting a model for Iran. Journal of Payavard Salamat. 2014; 8(2): 134-142.

26. Nikbakht M, Arastoo A, Ghasemzadeh R, Azizi A, Zahednejad $S$, Latifi SM. Developing a national minimum data set for physiotherapy in Iran: a delphi study. European Journal of Scientific Research. 2013; 107(4): 573-579.

27. Ghaneie M, Rezaie A, Ghorbani NR, Heidari RN, Arjomandi M, Zare M. Designing a minimum data set for breast cancer: a starting point for breast cancer registration in Iran. Iranian Journal of Public Health. 2013; 42(Suppl 1): 66-73.

28. Ahmadi M, Mohammadi A, Chraghbaigi R, Fathi T, Shojaee Baghini M. Iranian Red Crescent Medical Journal. 2014; 16(7).

29. Rafiei F, Ahmadi M, Hoseini F, Habibi Koolaei M. Nursing minimum data set. Iran Journal of Nursing. 2011; 24(71): 1927.

30. Kalankesh LR, Farahbakhsh M, Rahimi N. Data content and exchange in general practice: a review. Med Arch. 2014; 68(6): 414-418.

31. Carter J, Evans J, Tuttle M, Apelon TW. Making the "minimum data set" compliant with health information technology standards. 2006; Available from: http://aspe.hhs.gov/daltcp/reports/2006/MDS-HIT.htm.

32. WHO. Cancer Registration: Principles and Methods. International Agency for Research on Cancer; 1991.

33. Cystic Fibrosis Data Network. Cystic fibrosis data: meta-data and methodology. 2013; Available from: http://www.cysticfibrosisdata.org/metadata-and-methodology.htm.

34. Ministry of Health. Guideline for registration and classification of mortality causes. Tehran: Iranian Ministry of Health; 2013. 